\title{
Role of the angiopoietin/Tie system in pregnancy (Review)
}

\author{
D. KAPPOU ${ }^{1}$, S. SIFAKIS ${ }^{2}$, A. KONSTANTINIDOU ${ }^{3}$, N. PAPANTONIOU ${ }^{4}$ and D.A. SPANDIDOS $^{5}$ \\ ${ }^{1}$ First Department of Obstetrics and Gynecology, University of Athens, Athens; \\ ${ }^{2}$ Department of Obstetrics and Gynecology, University of Crete Medical School, Heraklion, Crete; \\ ${ }^{3}$ First Department of Pathology, University of Athens Medical School, ${ }^{4}$ Third Department of Obstetrics and Gynecology, \\ University of Athens, Athens; ${ }^{5}$ Laboratory of Clinical Virology, University of Crete Medical School, Heraklion, Crete, Greece
}

Received January 13, 2015; Accepted February 9, 2015

DOI: $10.3892 /$ etm.2015.2280

\begin{abstract}
Angiopoietin-1 and -2 are endogenous ligands for the vascular endothelium-specific receptor tyrosine kinase Tie-2. The angiopoietin/Tie system plays a critical role in the regulation of endothelial cell survival and vascular maturation and stability. Apart from its well-established role in vascular morphogenesis, emerging data support the involvement of angiopoietins in inflammation and various malignancies. Previous studies have underlined the significance of several angiogenic factors in normal placental development. In addition, angiogenic imbalance is observed in pregnancy complications related to impaired placentation, such as preeclampsia (PE) and intrauterine growth restriction (IUGR). However, there is only limited information available on the role of the angiopoietin/Tie system in the establishment of a competent feto-maternal vascular system. In this review, we present the current knowledge regarding the role of angiopoietins in normal pregnancy and pregnancy complications.
\end{abstract}

\section{Contents}

1. Introduction

2. The angiopoietin/Tie-2 system

3. The role of angiopoietins in normal pregnancy

4. Angiopoietins in pregnancy complications

5. Conclusion

\section{Introduction}

Successful placentation is dependent on the establishment of a competent vascular network formed by two processes: vasculo-

Correspondence to: Dr Stavros Sifakis, Department of Obstetrics and Gynecology, University of Crete Medical School, P.O. Box 2208, 71003 Heraklion, Crete, Greece

E-mail: stavros.sifakis@yahoo.com

Key words: angiopoietin-1, angiopoietin-2, angiopoietin/Tie system, intrauterine growth restriction, placenta, preeclampsia, pregnancy, vascular endothelium-specific receptor tyrosine kinase Tie-2 genesis, which involves the de novo formation of vessels from endothelial progenitor cells and branching and non-branching angiogenesis, which is the remodeling of the pre-existing vessels (1). The imbalance between pro-angiogenic and anti-angiogenic factors can lead to impaired placentation, causing major pregnancy complications, such as preeclampsia (PE) and intrauterine growth restriction (IUGR), which can lead to poor obstetric outcomes $(2,3)$. The role of various angiogenic factors in the pathophysiology of these conditions in pregnancy has been investigated (4). In recent years, in the field of angiogenesis research, studies have focused on the serum levels and placental expression of vascular endothelial growth factor (VEGF) and placental growth factor (PlGF) and its receptors in normal and pathological pregnancies $(5,6)$. However, there is only limited information available regarding the role of the angiopoietin/Tie signaling system in gestation, that is a second vascular endothelium-specific receptor tyrosine kinase pathway apart from the VEGF system (7). Since there is emerging evidence of the involvement of angiopoietins in pathologies characterized by vascular remodeling (8-10), such as sepsis, diabetic retinopathy and various malignancies, it would be of interest to explore the significance of these factors in the establishment of a competent feto-maternal vascular system that is essential for proper placental function and fetal growth.

In this review, we present current evidence of the function of angiopoietins and provide a detailed description of available data on the role of the angiopoietin/Tie pathway in normal placental development and fetal growth, as well as in pregnancy complications related to defective placentation, such as PE and IUGR.

\section{The angiopoietin/Tie system}

The angiopoietin system includes four ligands (Ang-1, Ang-2, Ang-3 and Ang-4), of which the most well characterized are Ang-1 and Ang-2, and two corresponding tyrosine kinase receptors (Tie-1 and Tie-2) $(11,12)$. During pregnancy, angiopoietins are mainly produced by the placenta and seem to play a critical role in endothelial cell survival and the remodeling of vessels (Fig. 1). In particular, these factors seem to act complementary to the VEGF system and contribute to the later stages of angiogenesis (12). Both Ang-1 and Ang-2 bind to Tie-2, an endothelial cell-specific tyrosine kinase receptor with similar affinity $(13,14)$. Although Ang-1 and Ang-2 share a similar 


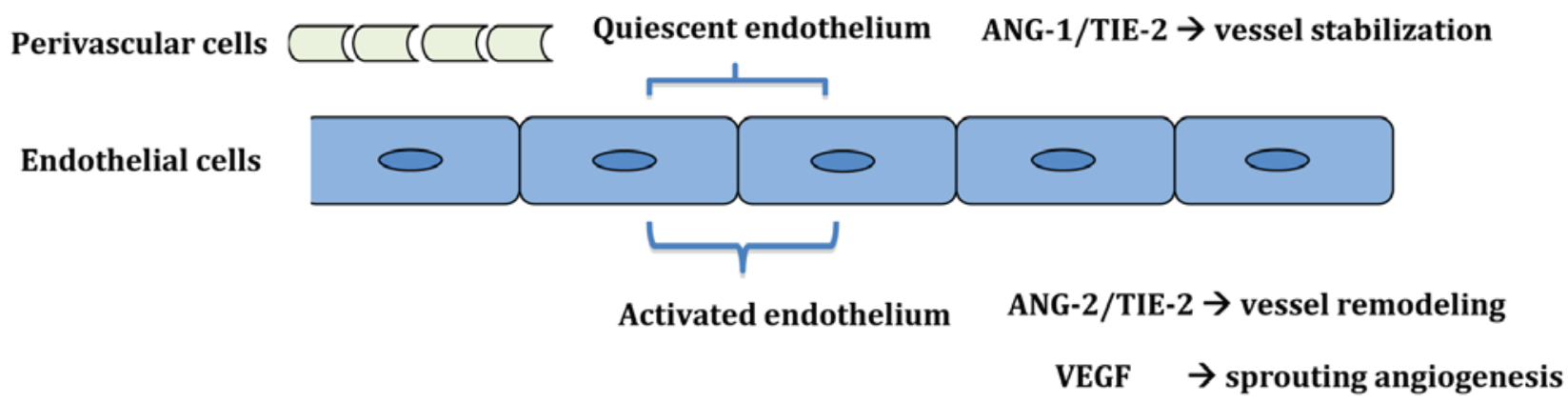

Figure 1. Role of the angiopoietin/Tie system in vessel remodeling and stabilization.

protein structure (Ang-2 is $60 \%$ homologous to Ang-1), their biological activities differ significantly (13). Ang-1 acts as a paracrine agonist to Tie-2, leads to receptor dimerization and induces its phosphorylation on several cytoplasmic residues to activate downstream signaling pathways, including the phosphoinositide 3 (PI3)-kinase/Akt and extracellular signalregulated kinase (ERK) pathways (15). The activation of the Akt pathway leads to the inhibition of FOXO transcription factors and downregulates the expression of Ang-2, endothelial cell-specific molecule 1 (ESM1) and PlGF (16). Apart from endothelial cells, previous studies have indicated that a distinct population of monocytes, known as Tie-2 expressing monocytes (TEM) and early hematopoietic cells also express the Tie-2 receptor $(17,18)$. The other known Tie receptor (Tie-1; tyrosine kinase with immunoglobin and epidermal growth factor homology domains) seems to have weak kinase activity and its functional role has not yet been fully elucidated (19). Ang-1 promotes the reorganization of endothelial cells and the structural integrity of blood vessels by recruiting and interacting with peri-endothelial cells $(14,19)$. An additional role of Ang-1 is to inhibit the activation of the vascular endothelial barrier and reduce the leakage and leucocyte migration into tissues induced by inflammatory agents (20). Despite the fact that the basic functioning of the pathway has been explored, there is no consistency as to the role of Ang-2 in certain conditions of pathological vascular remodeling, such as cancer and inflammation. Several lines of evidence suggest that Ang-2 binds to Tie-2, but primarily acts as an antagonist of Ang-1 signaling. In particular, Ang-2 disrupts the connections between the endothelium and perivascular cells and promotes cell death and vascular regression. In addition, Ang-2 renders endothelial cells more accessible to VEGF that promotes neovascularization $(13,19,21)$. However, in the case of insufficient angiogenic stimuli, Ang-2 causes endothelial cell apoptosis and vessel regression (13).

Challenging this predominant view of the antagonistic Ang-1/Ang-2 concept, there are data suggesting that with longer exposure or at higher concentrations, Ang-2 acts as a Tie-2 agonist in cultured endothelial cells $(22,23)$. In support of this view, in a previous study, when the Ang-2 gene was replaced with a cDNA encoding Ang-1 in Ang-2-deficient mice, the phenotype was partly rescued (24). Therefore, Ang-2 is currently considered as a context-dependent agonist or an antagonist of Tie-2. A recent study also stated that Ang-2 induced Tie-2-independent signaling by binding to integrins on endothelial cells that expressed low levels of Tie-2 and induced the phosphorylation of integrin adaptor protein, FAK, resulting in RAC1 activation (25). Furthermore, cellular experiments have demonstrated that Ang-2 modulates the responsiveness of endothelial cells to pro-inflammatory cytokines, such as tumor necrosis factor (TNF)- $\alpha$ and sensitizes these cells to an inflammatory response through an autocrine mechanism (26). Independently from their well-described role in the vascular endothelium, angiopoietins are also involved in the migration and proliferation of trophoblasts and the regulation of nitric oxide release during placentation (27).

Research interest has mainly been focused on the involvement of angiopoietins in various malignancies (28). In particular, the altered expression of Ang-1 and Ang-2 has been observed in several types of cancer, including ovarian and breast cancer, gastric carcinoma, melanoma and non-small cell lung cancer (28-30). In addition, recent studies producing encouraging results have suggested the potential use of specific Ang-2 inhibitors as anti-angiogenic agents to target the tumor vasculature, impairing its development and growth $(31,32)$.There is also accumulating evidence indicating that serum Ang-2 may also be a useful tool as a candidate prognostic biomarker for patients with certain types of cancer, such as colorectal cancer and melanoma $(33,34)$. The exact role of angiopoietins has also been broadly examined in other pathological conditions characterized by extensive vascular remodeling, such as diabetic retinopathy and sepsis $(8,10)$.

\section{The role of angiopoietins in normal pregnancy}

The first step towards the understanding of the mechanisms through which angiopoietins contribute to the development of pathological conditions in pregnancy is to explore the role of these molecules in normal pregnancy. Ang-1 and Ang-2 are both expressed in the placenta from the very early stages of pregnancy and they mediate a number of endothelial and non-endothelial effects that are thought to be pivotal for proper placental development $(27,35,36)$. Throughout gestation, the placental expression of Ang-1 normally increases, whereas that of Ang-2 and Tie-2 decreases $(35,36)$. The same trend has been demonstrated for the serum levels of these factors (37). The significance of these molecules in early placentation is also highlighted by the fact that the mRNA expression of Ang-2 during the first trimester was found to be 400 -fold higher than Ang-1 mRNA expression and 100-fold higher than 
VEGF mRNA expression, a factor which has an established role in placental angiogenesis (35). A possible explanation for the predominance of Ang-2 mRNA expression over that of the two angiogenic factors is that Ang-2 likely permits the fetal blood vessels to undergo remodeling and restructures the placenta vasculature to meet the increased oxygen and metabolic demands of the growing fetus. In addition, a previous cross-sectional study on 20 normal pregnancies at 8-41 weeks reported that the placental and protein expression of Ang-2 highly correlated and that they both markedly decreased toward term (35). During pregnancy, the placenta is most probably the main source of circulating Ang-2 as Ang-2 serum levels decrease rapidly following delivery (38).

A few previous in situ-hybridization studies have described the localization pattern of angiopoietins in the placenta in different stages of pregnancy. Seval et al (2008) demonstrated that in the very early human placenta (as early as the 4th week), Ang-1 protein was localized only in the syncytiotrophoblast, while Ang-2 was localized primarily in the syncytiotrophoblast, and to a lesser extent in the cytotrophoblastic layers of the placental villi (39). This is consistent with a previous report by Zhang et al (2001), who demonstrated that Ang-2 mRNA expression was readily detected in the syncytiotrophoblast in first trimester placenta (36). In the human placenta, high levels of Ang-2 mRNA expression have also been observed in perivascular cells and stromal macrophages in immature intermediate villi during the first and second trimester and in mature intermediate and terminal villi during the third trimester (35). Ang-1 is also weakly expressed in endothelial cells within intermediate villi, which is consistent with its role in promoting vascular maturation and stabilization (39). In a previous study, Dunk et al (2000) demonstrated that Ang-1 and Tie-2 were detected in the trophoblast bilayer of a first-trimester placenta, whereas Ang-2 mRNA was restricted to the cytotrophoblast (27). In the same study, Ang-1 and Ang-2 were shown to be implicated in the regulation of trophoblast behavior through different mechanisms and to promote the growth and migration of trophoblasts in vitro (27). In particular, Ang-1 acts as a potent chemotactic factor for trophoblasts, whereas Ang-2 enhances trophoblast DNA synthesis and triggers nitric oxide release (27). In term placentas, Ang-1 mRNA expression is restricted to the perivascular tissues of the primary stem villi, supporting its role in vessel maturation. Ang-2 is expressed throughout the term villous core and maintains the plasticity of placental vessels (27). In addition, Tie-2 receptors are also expressed in trophoblasts and participate in the regulation of trophoblast behavior (27). The inconsistency observed between different studies may be due to the different gestational age at placental tissue sampling and the different methodologies used.

Experimental studies have also shed further light on the key role of angiopoietins in fetal viability and wellness. In a previous study, mice with null mutations in the Ang-1 gene died at day 12.5 of pregnancy and showed severe vascular abnormalities characterized by disturbance in endothelial/pericyte interactions (40). In another study, mice genetically deficient for Ang-2 showed complex lymphatic and vascular defects and the majority of the mice died two weeks post-natally (24). In turn, Geva et al (2005) used a novel murine model system and showed that in utero Ang-2 gene delivery impaired endothelial cell adhesiveness, leading to vascular leakiness with perivascular edema (41). Mice null for Tie-2 have been shown to exhibit severe vascular damage and cardiac abnormalities that cause embryonic lethality at approximately embryonic day 10.5 (42). A previous study by Wulff et al (2002) provided a detailed description of the spatial and temporal expression of Ang-1, Ang-2 and Tie-2 in the primate placenta and indicated that Ang-1/Tie-2 may support feto-placental vascular development and stabilization, whereas Ang-2/Tie-2 may remodel the maternal vasculature (43).

\section{Angiopoietins in pregnancy complications}

The majority of published studies on the role of angiogenic factors in the development of pregnancy disorders related to impaired placentation, such as PE and IUGR have focused on the expression profiles of VEGF, PIGF and its receptors. However, few studies have examined the expression pattern of angiopoietins in the above-mentioned pregnancy disorders.

PE affects approximately $2-7 \%$ of all pregnancies and is a major cause of maternal and perinatal morbidity and mortality (44). Although the pathogenesis of this condition is multifactorial, there is robust evidence indicating that PE is associated with impaired placentation and placental hypoxia related to an imbalance between pro-angiogenic and antiangiogenic factors $(4,45)$. Previous studies that have examined the placental expression of angiopoietins in pregnancies complicated by PE have presented conflicting results and have used rather small sample sizes (Table I). Zhang et al (2001) examined the placental tissue samples from nine women with PE at 31-40 weeks of pregnancy and demonstrated lower levels of Ang-2 mRNA expression compared to the control group (36). A similar study by Geva et al (2002) demonstrated that the placental Ang-1 and Tie-2 mRNA expression was not altered in five cases of PE with IUGR in the third trimester, whereas there was an increase in Ang-2 mRNA expression that did not reach statistical significance (35). In addition, Sung et al (2011) revealed no marked changes in the placental expression of Ang-1, Ang-2 and Tie-2 in placental samples from 19 preeclamptic pregnancies (46). In a recent study, the expression of Ang-2 was significantly increased in placentas obtained from pregnancies complicated by severe PE, whereas no change was observed for Ang-1 levels (47). In addition, no significant association was observed between Ang-1 and Ang-2 in the whole study group. However, the aforementioned study used a small study sample and the proportion of IUGR fetuses among the PE group was not reported. Similarly, a recent study by our group demonstrated an increase in the expression of Ang-2 in the PE and PE-IUGR group consisting of 58 pregnant women compared to the control group (48). To the best of our knowledge, this is the largest sample size in similar studies reported thus far. Our data also indicated that Ang-2 expression was even higher in the PE-IUGR group, suggesting a direct association of this angiogenic factor with the severity of the disease. Additionally, we detected the decreased expression of Ang-1 in $\mathrm{PE}$, a trend that was not observed in the study by Han et al (47). Our analysis revealed a weak association between Ang-1 and Ang-2 in the PE-IUGR group and no significant correlation was observed between these parameters in the control and the PE group, in accordance with the study by Han et al. These findings are in line with those of earlier reports that support 
Table I. List of studies that examined the levels of Ang-1, Ang-2 and Tie-2 in placental specimens from pregnancies complicated by PE and IUGR.

\begin{tabular}{|c|c|c|c|c|c|c|c|}
\hline Authors/(Refs.) & Year & $\begin{array}{l}\text { Sample } \\
\text { size }\end{array}$ & $\begin{array}{c}\text { Gestational } \\
\text { frame in weeks }\end{array}$ & $\begin{array}{l}\text { Pregnancy } \\
\text { complication }\end{array}$ & Ang-1 & Ang-2 & Tie-2 \\
\hline Dunk et al (27) & 2000 & 6 & $28-36$ & Severe IUGR & & NS & \\
\hline Zhang et al (36) & 2001 & 9 & $31-40$ & $\mathrm{PE}$ & & $\downarrow$ & \\
\hline Geva et al (35) & 2002 & 5 & $25-41$ & Severe PE & & NS & \\
\hline Sung et al (46) & 2011 & 19 & & $\mathrm{PE}$ & NS & NS & NS \\
\hline Han et al (47) & 2012 & 16 & $28-41$ & Severe PE & NS & $\uparrow$ & \\
\hline Kappou et al (48) & 2014 & $\begin{array}{c}28 \mathrm{PE}+ \\
30 \mathrm{PE} / \mathrm{IUGR}\end{array}$ & $34-39$ & $\begin{array}{c}\text { PE } \\
\text { PE + IUGR }\end{array}$ & $\downarrow$ & $\uparrow$ & $\uparrow$ \\
\hline
\end{tabular}

PE, preeclampsia; IUGR, intrauterine growth restriction; NS, not significant; The upward arrows indicate the upregulated expression and the downward arrows indicate the downregulated expression of Ang-1, Ang-2 and Tie-2 in the indicated studies.

an excess of circulating levels of anti-angiogenic factors even before the clinical manifestation of PE $(2,49)$. It is notable that Zhang et al (2001) examined how the oxygen tension in the placenta can influence the levels of angiopoietin expression and demonstrated that the Ang-1 mRNA level was not significantly altered by reduced oxygen, while a reduction in oxygen tension significantly increased the levels of Ang-2 mRNA through the activation of hypoxia-inducible factor-1 $\alpha$, leading to vessel instability and remodeling (36). This is important, as placenta hypoxia is an established factor of PE pathogenesis. Consistently, data from s study on bovine models demonstrated that hypoxia upregulated the expression of of Ang-2, but not Ang-1 mRNA expression (50).

The same inconsistency is also apparent in studies that have examined the maternal circulation levels of angiopoietins in pregnancies complicated by PE before or after the clinical manifestation of the disease. In cases with established PE, the majority of studies have reported lower circulating concentrations of Ang-2 $(38,51,52)$ and Tie- $2(46,51,53)$ compared to the unaffected controls. However, Leinonen et al demonstrated that circulating maternal Ang-2 concentrations were elevated in the early midtrimester (16-20 weeks of gestation) in women that subsequently developed PE (37). These findings support the hypothesis that an excess of anti-angiogenic factors may be a predisposing factor for PE and may be apparent before the clinical onset of the disease. Moreover, the authors stated that the levels of Ang-2 were associated with the severity of the disease. Similarly, our recently published data demonstrated a higher placental expression of Ang-2 in pregnancies complicated by PE and IUGR, which is a distinct and more severe form of PE (48). A recent longitudinal study demonstrated the upregulation of Ang-2 levels and decreased Ang-1 levels in maternal serum in the whole gestational frame; in addition it was shown that the Ang-1/Ang-2 ratio at 25-28 gestational weeks may have predictive value for women who later developed PE (54). This is a very promising finding, as there is evidence supporting the ability of other angiogenic factors, such as PIGF to identify pathological pregnancy outcomes, such as IUGR and PE before clinical diagnosis with adequate sensitivity $(55,56)$. At 11-13 weeks of gestation, the maternal serum concentration of Ang-2 has been reported to be either lower (57) or not altered in women who subsequently develop PE (58). Akolekar et al concomitantly examined the first trimester maternal serum levels of Ang-2 in pregnancies with gestational hypertension and found no significant changes compared to the control group (58). A recent study assessed Ang-1, Ang-2 and the Ang-1/Ang-2 ratio levels in the first trimester of pregnancy and the association with adverse pregnancy outcomes (small for gestational age, pre-term birth, PE, miscarriage after 10 weeks of gestation, and stillbirth) (59). According to the findings of the former study, low Ang-2 levels and a high Ang-1/Ang-2 ratio were related to an increased risk for most adverse pregnancy outcomes, but did not improve the prediction given by maternal and clinical factors alone (58). In support of this finding, first trimester maternal serum levels of Ang-1 were found to be elevated in women with PE and concomitant IUGR (37). As regards the studies on Tie-2 in maternal serum, it should be clarified that the measurable part is the circulating soluble Tie-2 receptor (sTie-2) fragment that is cleaved constitutively from the extracellular domain of membrane-bound Tie-2, binds both to Ang-1 and Ang-2 and inhibits angiopoietin-mediated Tie-2 activation. Moreover, data have suggested the existence of a novel metalloproteinase-dependent mechanism through which release of sTie- 2 is induced by VEGF in endometrial endothelial cells and leads to a decrease in active receptors on the cell surface (60). As VEGF and Ang act through distinct tyrosine kinase receptors, this mechanism suggests that VEGF is involved in the regulation of Ang function indirectly through the proteolytic shedding of Tie-2.

The role of angiopoietins has only scarcely been investigated in pregnancies with fetal growth restriction, a condition that complicates $4-7 \%$ of live births in developed countries and is related to a spectrum of perinatal complications, including fetal morbidity and mortality. Placental insufficiency related to defective placental angiogenesis often represents the underlying cause of IUGR (61). In particular, placentas from pregnancies complicated IUGR are characterized by defective vascular transformation and terminal villus formation (3). At 11-13 weeks of gestation, Wang et al demonstrated that Ang-2 maternal serum levels were reduced in 13 women who subsequently developed 
IUGR compared to 23 unaffected controls (57). However, in that study, it was not defined whether IUGR was related to PE or could be classified as idiopathic. This is in contrast to the study by Leinonen et al that examined the circulating levels of Ang-1, Ang-2 and Tie-2 at 12-15 or 16-20 gestational weeks in 16 women with subsequent isolated IUGR compared to 59 healthy women served as controls (37). In that study, maternal serum Ang-2 concentrations were higher in women with subsequent IUGR only at 16-20 gestational weeks. Further studies are required to elucidate whether the involvement of these factors is a primary mechanism leading to the abnormal placental development or whether the altered expression of these angiogenic growth factors reflects other, as yet unidentified mechanisms. The altered placental expression of the angiopoietin/Tie-2 system has been observed in pregnancies complicated by placenta accreta and in women with recurrent abortion $(62,63)$; however, these conditions are not within the scope of this review article.

\section{Conclusion}

There is emerging evidence indicating that the angiopoietin/Tie-2 signaling system is essential for the proper reorganization of the placental vascular system even from the very first stages of pregnancy. In recent years, an increasing number of studies in the field of cancer research have not only highlighted the important role of angiopoietins in tumor progression, but have also attempted to implicate them as factors in the prognostic and therapeutic field $(28,30,64,65)$. However, available information on the function of angiopoietins during pregnancy is still limited, as the main interest has been focused on the VEGF and PIGF systems. In addition, the majority of published studies are case-control studies that describe the observed alterations in the levels of angiopoietins in pathological pregnancies, but do not elucidate the aetiopathogenesis and the underlying mechanisms. Additional studies with sufficient sample sizes and strict criteria for the recruitment of study populations are required in order to draw a final conclusion as to the alteration profile of angiopoietins in pregnancies complicated by PE and/or IUGR. Moreover, the prognostic value of these factors as biomarkers for particular pathological conditions during pregnancy should also be investigated in prospective studies.

\section{References}

1. Yancopoulos GD, Davis S, Gale NW, Rudge JS, Wiegand SJ and Holash J: Vascular-specific growth factors and blood vessel formation. Nature 407: 242-248, 2000.

2. Maynard SE and Karumanchi SA: Angiogenic factors and preeclampsia. Semin Nephrol 31: 33-46, 2011.

3. Gourvas V, Dalpa E, Konstantinidou A, Vrachnis N, Spandidos DA and Sifakis S: Angiogenic factors in placentas from pregnancies complicated by fetal growth restriction (review). Mol Med Rep 6: 23-27, 2012.

4. Chaiworapongsa T, Romero R, Korzeniewski SJ, et al: Maternal plasma concentrations of angiogenic/antiangiogenic factors in the third trimester of pregnancy to identify the patient at risk for stillbirth at or near term and severe late preeclampsia. Am J Obstet Gynecol 208: 287.e1-287.e15, 2013.

5. Gómez-Arriaga PI, Herraiz I, López-Jiménez EA, Escribano D, Denk B and Galindo A: Uterine artery Doppler and sFlt-1/PIGF ratio: Prognostic value in early-onset pre-eclampsia. Ultrasound Obstet Gynecol 43: 525-532, 2014.
6. Andraweera PH, Dekker GA and Roberts CT: The vascular endothelial growth factor family in adverse pregnancy outcomes. Hum Reprod Update 18: 436-457, 2012.

7. Charnock-Jones DS: Soluble flt-1 and the angiopoietins in the development and regulation of placental vasculature. J Anat 200: 607-615, 2002.

8. Park SW, Yun JH, Kim JH, Kim KW, Cho CH and Kim JH: Angiopoietin 2 induces pericyte apoptosis via $\alpha 3 \beta 1$ integrin signaling in diabetic retinopathy. Diabetes 63: 3057-3068, 2014.

9. Saharinen P, Eklund L, Pulkki K, Bono P and Alitalo K: VEGF and angiopoietin signaling in tumor angiogenesis and metastasis. Trends Mol Med 17: 347-362, 2011.

10. Mussap M, Cibecchini F, Noto A and Fanos V: In search of biomarkers for diagnosing and managing neonatal sepsis: The role of angiopoietins. J Matern Fetal Neonatal Med 26 (Suppl 2): 24-26, 2013.

11. Thurston G: Role of Angiopoietins and Tie receptor tyrosine kinases in angiogenesis and lymphangiogenesis. Cell Tissue Res 314: 61-68, 2003.

12. Thomas $\mathbf{M}$ and Augustin HG: The role of the Angiopoietins in vascular morphogenesis. Angiogenesis 12: 125-137, 2009.

13. Maisonpierre PC, Suri C, Jones PF, et al: Angiopoietin-2, a natural antagonist for Tie2 that disrupts in vivo angiogenesis. Science 277: 55-60, 1997.

14. Davis S, Aldrich TH, Jones PF, et al: Isolation of angiopoietin-1, a ligand for the TIE2 receptor, by secretion-trap expression cloning. Cell 87: 1161-1169, 1996.

15. Khan AA, Sandhya VK, Singh P, et al: Signalling network map of endothelial TEK tyrosine kinase. J Signal Transduct 2014: 173026, 2014.

16. Daly C, Wong V, Burova E, et al: Angiopoietin-1 modulates endothelial cell function and gene expression via the transcription factor FKHR (FOXO1). Genes Dev 18: 1060-1071, 2004.

17. Coffelt SB, Tal AO, Scholz A, et al: Angiopoietin-2 regulates gene expression in TIE2-expressing monocytes and augments their inherent proangiogenic functions. Cancer Res 70: 5270-5280, 2010.

18. Yuan HT, Venkatesha S, Chan B, Deutsch U, Mammoto T, Sukhatme VP, Woolf AS and Karumanchi SA: Activation of the orphan endothelial receptor Tie1 modifies Tie2-mediated intracellular signaling and cell survival. FASEB J 21: 3171-3183, 2007.

19. Augustin HG, Koh GY, Thurston G and Alitalo K: Control of vascular morphogenesis and homeostasis through the angiopoietin-Tie system. Nat Rev Mol Cell Biol 10: 165-177, 2009.

20. Thurston G, Rudge JS, Ioffe E, Zhou H, Ross L, Croll SD, Glazer N, Holash J, McDonald DM and Yancopoulos GD: Angiopoietin-1 protects the adult vasculature against plasma leakage. Nat Med 6: 460-463, 2000.

21. Scharpfenecker M, Fiedler U, Reiss Y and Augustin HG: The Tie-2 ligand angiopoietin-2 destabilizes quiescent endothelium through an internal autocrine loop mechanism. J Cell Sci 118: 771-780, 2005.

22. Daly C, Eichten A, Castanaro C, et al: Angiopoietin-2 functions as a Tie2 agonist in tumor models, where it limits the effects of VEGF inhibition. Cancer Res 73: 108-118, 2013.

23. Teichert-Kuliszewska K, Maisonpierre PC,Jones N, Campbell AI, Master Z, Bendeck MP, Alitalo K, Dumont DJ, Yancopoulos GD and Stewart DJ: Biological action of angiopoietin-2 in a fibrin matrix model of angiogenesis is associated with activation of Tie2. Cardiovasc Res 49: 659-670, 2001.

24. Gale NW, Thurston G, Hackett SF, Renard R, Wang Q, McClain J, Martin C, Witte C, Witte MH and Jackson D: Angiopoietin-2 is required for postnatal angiogenesis and lymphatic patterning, and only the latter role is rescued by Angiopoietin-1. Dev Cell 3: 411-423, 2002.

25. Felcht M, Luck R, Schering A, et al: Angiopoietin-2 differentially regulates angiogenesis through TIE2 and integrin signaling. J Clin Invest 122: 1991-2005, 2012.

26. Fiedler U, Reiss Y, Scharpfenecker M, et al: Angiopoietin-2 sensitizes endothelial cells to TNF-alpha and has a crucial role in the induction of inflammation. Nat Med 12: 235-239, 2006.

27. Dunk C, Shams M, Nijjar S, Rhaman M, Qiu Y, Bussolati B and Ahmed A: Angiopoietin-1 and angiopoietin-2 activate trophoblast Tie-2 to promote growth and migration during placental development. Am J Pathol 156: 2185-2199, 2000.

28. Shim WS, Ho IA and Wong PE: Angiopoietin: A TIE(d) balance in tumor angiogenesis. Mol Cancer Res 5: 655-665, 2007.

29. Brunckhorst MK, Xu Y, Lu R and Yu Q: Angiopoietins promote ovarian cancer progression by establishing a procancer microenvironment. Am J Pathol 184: 2285-2296, 2014. 
30. Huang $\mathrm{H}$, Bhat $\mathrm{A}$, Woodnutt $\mathrm{G}$ and Lappe R: Targeting the ANGPT-TIE2 pathway in malignancy. Nat Rev Cancer 10: 575-585, 2010.

31. Biel NM and Siemann DW: Targeting the Angiopoietin-2/Tie-2 axis in conjunction with VEGF signal interference. Cancer Lett: Oct 12, 2014 (Epub ahead of print).

32. Mazzieri R, Pucci F, Moi D, et al: Targeting the ANG2/TIE2 axis inhibits tumor growth and metastasis by impairing angiogenesis and disabling rebounds of proangiogenic myeloid cells. Cancer Cell 19: 512-526, 2011.

33. Goede V, Coutelle O, Neuneier J, et al: Identification of serum angiopoietin-2 as a biomarker for clinical outcome of colorectal cancer patients treated with bevacizumab-containing therapy. Br J Cancer 103: 1407-1414, 2010.

34. Helfrich I, Edler L, Sucker A, et al: Angiopoietin-2 levels are associated with disease progression in metastatic malignant melanoma. Clin Cancer Res 15: 1384-1392, 2009.

35. Geva E, Ginzinger DG, Zaloudek CJ, Moore DH, Byrne A and Jaffe RB: Human placental vascular development: Vasculogenic and angiogenic (branching and nonbranching) transformation is regulated by vascular endothelial growth factor-A, angiopoietin-1, and angiopoietin-2. J Clin Endocrinol Metab 87: 4213-4224, 2002.

36. Zhang EG, Smith SK, Baker PN and Charnock-Jones DS: The regulation and localization of angiopoietin-1, -2, and their receptor Tie2 in normal and pathologic human placentae. Mol Med 7: 624-635, 2001.

37. Leinonen E, Wathén KA, Alfthan H, Ylikorkala O, Andersson S, Stenman UH and Vuorela P: Maternal serum angiopoietin-1 and -2 and tie-2 in early pregnancy ending in preeclampsia or intrauterine growth retardation. J Clin Endocrinol Metab 95: $126-133,2010$.

38. Hirokoshi K, Maeshima Y, Kobayashi K, Matsuura E, Sugiyama H, Yamasaki Y, Masuyama H, Hiramatsu Y and Makino H: Elevated serum sFlt-1/Ang-2 ratio in women with preeclampsia. Nephron Clin Pract 106: c43-c50, 2007.

39. Seval Y, Sati L, Celik-Ozenci C, Taskin O and Demir R: The distribution of angiopoietin-1, angiopoietin-2 and their receptors tie- 1 and tie- 2 in the very early human placenta. Placenta 29: 809-815, 2008.

40. Suri C, Jones PF, Patan S, Bartunkova S, Maisonpierre PC, Davis S, Sato TN and Yancopoulos GD: Requisite role of angiopoietin-1, a ligand for the TIE2 receptor, during embryonic angiogenesis. Cell 87: 1171-1180, 1996.

41. Geva E, Ginzinger DG, Moore DH II, Ursell PC and Jaffe RB: In utero angiopoietin-2 gene delivery remodels placental blood vessel phenotype: A murine model for studying placental angiogenesis. Mol Hum Reprod 11: 253-260, 2005.

42. Sato TN, Tozawa Y, Deutsch U, Wolburg-Buchholz K, Fujiwara Y, Gendron-Maguire M, Gridley T, Wolburg H, Risau W and Qin Y: Distinct roles of the receptor tyrosine kinases Tie-1 and Tie-2 in blood vessel formation. Nature 376: 70-74, 1995.

43. Wulff C, Wilson H, Dickson SE, Wiegand SJ and Fraser HM: Hemochorial placentation in the primate: Expression of vascular endothelial growth factor, angiopoietins, and their receptors throughout pregnancy. Biol Reprod 66: 802-812, 2002.

44. Steegers EA, von Dadelszen P, Duvekot JJ and Pijnenborg R: Pre-eclampsia. Lancet 376: 631-644, 2010.

45. Kleinrouweler CE, Wiegerinck MM, Ris-Stalpers C, Bossuyt PM, van der Post JA, von Dadelszen P, Mol BW and Pajkrt E; EBM CONNECT Collaboration: Accuracy of circulating placental growth factor, vascular endothelial growth factor, soluble fms-like tyrosine kinase 1 and soluble endoglin in the prediction of pre-eclampsia: A systematic review and meta-analysis. BJOG 119: 778-787, 2012.

46. Sung JF, Fan X, Dhal S, Dwyer BK, Jafari A, El-Sayed YY, Druzin ML and Nayak NR: Decreased circulating soluble Tie2 levels in preeclampsia may result from inhibition of vascular endothelial growth factor (VEGF) signaling. J Clin Endocrinol Metab 96: E1148-E1152, 2011.

47. Han SY, Jun JK, Lee CH, Park JS and Syn HC: Angiopoietin-2: A promising indicator for the occurrence of severe preeclampsia. Hypertens Pregnancy 31: 189-199, 2012.
48. Kappou D, Sifakis S, Androutsopoulos V, Konstantinidou A, Spandidos DA and Papantoniou N: Placental mRNA expression of angiopoietins (Ang)-1, Ang-2 and their receptor Tie-2 is altered in pregnancies complicated by preeclampsia. Placenta 35 : 718-723, 2014.

49. Szpera-Gozdziewicz A and Breborowicz GH: Endothelial dysfunction in the pathogenesis of pre-eclampsia. Front Biosci (Landmark Ed) 19: 734-746, 2014.

50. Oh H, Takagi H, Suzuma K, Otani A, Matsumura M and Honda Y: Hypoxia and vascular endothelial growth factor selectively up-regulate angiopoietin- 2 in bovine microvascular endothelial cells. J Biol Chem 274: 15732-15739, 1999.

51. Vuorela P, Matikainen MT, Kuusela P, Ylikorkala O, Alitalo K and Halmesmäki E: Endothelial tie receptor antigen in maternal and cord blood of healthy and preeclamptic subjects. Obstet Gynecol 92: 179-183, 1998.

52. Hirokoshi K, Maeshima Y, Kobayashi K, Matsuura E, Sugiyama H, Yamasaki Y, Masuyama H, Hiramatsu Y and Makino H: Increase of serum angiopoietin-2 during pregnancy is suppressed in women with preeclampsia. Am J Hypertens 18: 1181-1188, 2005.

53. Gotsch F, Romero R, Kusanovic JP, et al: Preeclampsia and small-for-gestational age are associated with decreased concentrations of a factor involved in angiogenesis: Soluble Tie-2. J Matern Fetal Neonatal Med 21: 389-402, 2008.

54. Bolin M, Wiberg-Itzel E, Wikström AK, Goop M, Larsson A, Olovsson $\mathrm{M}$ and Akerud $\mathrm{H}$ : Angiopoietin-1/angiopoietin-2 ratio for prediction of preeclampsia. Am J Hypertens 22: 891-895, 2009.

55. Schoofs K, Grittner U, Engels T, Pape J, Denk B, Henrich W and Verlohren S: The importance of repeated measurements of the sFlt-1/PlGF ratio for the prediction of preeclampsia and intrauterine growth restriction. J Perinat Med 42: 61-68, 2014.

56. Vrachnis N, Kalampokas E, Sifakis S, Vitoratos N, Kalampokas T, Botsis D and Iliodromiti Z: Placental growth factor (PlGF): A key to optimizing fetal growth. J Matern Fetal Neonatal Med 26: 995-1002, 2013.

57. Wang Y, Tasevski V, Wallace EM, Gallery ED and Morris JM: Reduced maternal serum concentrations of angiopoietin-2 in the first trimester precede intrauterine growth restriction associated with placental insufficiency. BJOG 114: 1427-1431, 2007.

58. Akolekar R, Casagrandi D, Skyfta E, Ahmed AA and Nicolaides KH: Maternal serum angiopoietin-2 at 11 to 13 weeks of gestation in hypertensive disorders of pregnancy. Prenat Diagn 29: 847-851, 2009.

59. Schneuer FJ, Roberts CL, Ashton AW, Guilbert C, Tasevski V, Morris JM and Nassar N: Angiopoietin 1 and 2 serum concentrations in first trimester of pregnancy as biomarkers of adverse pregnancy outcomes. Am J Obstet Gynecol 210: 345.e1-e9, 2014.

60. Findley CM, Cudmore MJ, Ahmed A and Kontos CD: VEGF induces Tie2 shedding via a phosphoinositide 3-kinase/Akt dependent pathway to modulate Tie2 signaling. Arterioscler Thromb Vasc Biol 27: 2619-2626, 2007.

61. Regnault TR, Galan HL, Parker TA and Anthony RV: Placental development in normal and compromised pregnancies- a review. Placenta 23 (Suppl A): S119-S129, 2002.

62. Tseng JJ, Hsu SL, Ho ES, Hsieh YT, Wen MC and Chou MM: Differential expression of angiopoietin-1, angiopoietin-2, and Tie receptors in placentas from pregnancies complicated by placenta accreta. Am J Obstet Gynecol 194: 564-571, 2006.

63. Vuorela P, Carpén O, Tulppala M and Halmesmäki E: VEGF, its receptors and the tie receptors in recurrent miscarriage. Mol Hum Reprod 6: 276-282, 2000.

64. Hall M, Gourley C, McNeish I, Ledermann J, Gore M, Jayson G, Perren T, Rustin G and Kaye S: Targeted anti-vascular therapies for ovarian cancer: current evidence. Br J Cancer 108: 250-258, 2013.

65. Gerald D, Chintharlapalli S, Augustin HG and Benjamin LE: Angiopoietin-2: an attractive target for improved antiangiogenic tumor therapy. Cancer Res 73: 1649-1657, 2013. 